\title{
Letalidade na epidemiologia da doença meningocócica: estudo na região de Campinas, SP, 1993 a 1998
}

Case fatality rate for meningococcal disease: study in the region of Campinas, SP, Brazil 1993 to 1998

Maria Rita C Donalisioa, Brigina Kemp ${ }^{b}$, Marilu MM Rochac e Raquel MF Ramalheirad $^{d}$

aDepartamento de Medicina Preventiva e Social da Faculdade de Ciências M édicas da U niversidade Estadual de Campinas. Campinas, SP, Brasil. ' Vigilância Epidemiológica DIR XII - Campinas da Secretaria da Saúde do Estado de São Paulo. Campinas, SP, Brasil. 'Instituto Adolfo Lut de Campinas. Campinas, SP, Brasil 


\title{
Letalidade na epidemiologia da doença meningocócica: estudo na região de Campinas, SP, 1993 a 1998 Case fatality rate for meningococcal disease: study in the region of Campinas, SP, Brazil 1993 to 1998
}

\author{
Maria Rita C Donalisioa, Brigina Kemp ${ }^{b}$, Marilu MM Rochac e Raquel MF Ramalheirad \\ ${ }^{\text {aD }}$ epartamento de Medicina Preventiva e Social da Faculdade de Ciências Médicas da U niversidade \\ Estadual de Campinas. Campinas, SP, Brasil. 'Vigilância Epidemiológica DIR XII - Campinas da \\ Secretaria da Saúde do Estado de São Paulo. Campinas, SP, Brasil. Instituto Adolfo Lut de Campinas. \\ Campinas, SP, Brasil
}

\section{Descritores}

Infecções meningocócicas". Mortalidade". Vigilância epidemiológica\# ${ }^{\#}$ Neisseria meningitidis". Infecções meningocócicas, epidemiologia. Fatores de risco. - Coeficiente de letalidade.

\section{Resumo}

Objetivo

Analisar a tendência da letalidade e da incidência da doença meningocócica no período de 1993 a 1998 na região de Campinas, SP, abrangendo cinco municípios de seu entorno (1,2 milhões de habitantes).

Métodos

Foi realizado estudo longitudinal retrospectivo de todos os casos notificados (375) da doença meningocócica pela vigilância epidemiológica regional. Por meio de análise de regressão logística foram identificados os fatores associados ao aumento da letalidade dessa doença.

Resultados

Os anos de 1996 e de 1997 apresentaram maiores coeficientes de letalidade $(23,8 \%)$, coincidindo com picos de incidência do sorogrupo B, altos percentuais de meningococcemia e menor investigação etiológica. Observou-se padrão sazonal e predomínio da circulação da Neisseria meningitidis das cepas B:4:P1.15 e $\mathrm{C}: 2 \mathrm{~b}: \mathrm{P} 1.3$. Os fatores relacionados com o aumento da letalidade pela análise de regressão logística foram: presença de meningococcemia, com ou sem meningite (odds ratio ajustado $\left(\mathrm{OR}_{\mathrm{aj}}\right)$ 13,88 e intervalo de confiança de $95 \%$ (IC) 4,6842,13); idade acima de 30 anos $\left(\mathrm{OR}_{\mathrm{aj}} 6,42\right.$; IC 2,32-17,80); idade inferior a 1 ano $\left(\mathrm{OR}_{\mathrm{aj}}\right.$ 2,95; IC 1,55-5,63); e sorogrupo B (OR ${ }_{\mathrm{aj}} 2,33$; IC 1,14- 4,79).

Conclusões

A septicemia, a idade e o sorogrupo mostraram-se variáveis preditoras de morte. Em alguns anos os coeficientes de letalidade apresentaram-se altos, indicando a necessidade de investigação da qualidade e da agilidade da assistência à saúde na prevenção dos óbitos. O percentual de identificação etiológica dos casos dificultou conclusões mais precisas sobre o comportamento epidemiológico das cepas.
Correspondência para/Correspondence to: Maria Rita C. Donalisio

Faculdade de Ciências Médicas da Unicamp Caixa Postal 6111

13083-970 Campinas, SP, Brasil

E-mail: donalis@dglnet.com.br
Edição subvencionada pela Fapesp (Processo no 00/01601-8).

Recebido em 4/11/1999. Reapresentado em 28/10/2000. A provado em 3/11/2000. 


\section{Keywords}

Meningococcal infections . $^{\#}$. Mortality". Epidemiologic surveillance ${ }^{\#}$. Neisseria meningitidis ${ }^{\#}$. Meningococcal infections, epidemiology. Risk factors. - Fatality rate.

\begin{abstract}
Objective

To analyze the trends of the meningococcal disease cumulative incidence and casefatality rate in the region of Campinas, Brazil, an area that encircles five cities and 1.2 million inhabitants, from 1993 to 1998.

\section{Methods}

A longitudinal retrospective study of all case records (375) obtained from the regional epidemiological surveillance system was carried out. A logistic regression analysis allowed identifying the risk factors related to fatal outcomes of meningococcal disease.

Results

The highest fatality rates (23.8\%) were seen in the period of 1996 and 1997, coinciding with the incidence peaks of serogroup $B$ Neisseria and a high percentage of septicemia cases. Also at the same period there was registered a poor etiological investigation of the cases. A seasonal pattern and the predomination of strains B:4:P1.15 and $C: 2 b: P 1.3$ were observed. In the logistic regression analysis, the risk factors related to fatality were: septicemia with or without meningitis (adjusted odds ratio $\left[O R_{a}\right]=$ 13.88 and $95 \%$ confidence interval $[C I]=4.68-42.13)$; age over 30 years $\left(O R_{a}=\right.$ 6.42; $C I=2.32-17.80) ;$ age under 1 year $\left(O R_{a}=2.95 ; C I=1.55-5.63\right) ;$ and serogroup $B\left(O R_{a}=2.33 ; C I=1.14-4.79\right)$.

\section{Conclusions}

Septicemia, age and serogroup were predictive variables related to a fatal outcome. In 1996 and 1997, case-fatality rates were high, indicating the need to further assessment of the quality of the services delivered and their readiness to take preventive action. The lack of etiological identification in many cases precluded more accurate inferences about the epidemiological behavior of Neisseria meningitidis in the region.
\end{abstract}

\section{INTRO DU ÇÃO}

A doença meningocócica (DM) tem sido importante causa de morbidade e de mortalidade no Estado de São Paulo. Desde o final dos anos 80 , há registros de aumento nos coeficientes de incidência, sendo que na Grande São Paulo ficou caracterizada uma epidemia a partir de 1988. Nesse período houve predomínio da circulação da Neisseria meningitidis sorogrupo B, observando-se no início da década de 90 um aumento na proporção dos casos pelo sorogrupo C. Comportamento semelhante ao do Estado, no mesmo período, pôde ser verificado no município de Campinas e região. ${ }^{2,7}$ De um modo geral, na década de 80 , grande parte dos casos de DM registrados nos países escandinavos e americanos foram causados pelo meningococo sorogrupo B. ${ }^{6,12}$

Na região de Campinas foram observados aumento gradual da incidência da DM até meados de 1990 e alta letalidade, particularmente nos anos de 1996 e de 1997, o que exigiu investigação mais apurada. Estudos têm demonstrado a importância do coeficiente de letalidade como um indicador fundamental para a análise do comportamento epidemiológico da DM. ${ }^{6,15,16}$

O objetivo do presente trabalho é analisar a tendência da DM no período de 1993 a 1998, na região de Campinas, identificar fatores que possam estar relacionados com a letalidade e subsidiar o sistema de vigilância epidemiológica regional para intervenções específicas no controle da doença e do óbito.

\section{MÉTODOS}

Trata-se de estudo longitudinal retrospectivo da letalidade e de características epidemiológicas dos casos (375) de DM notificados pela vigilância epidemiológica na região de Campinas, SP, nos anos de 1993 a 1998

A área delimitada para o estudo foi o município de Campinas e outros quatro municípios no seu entorno: Sumaré, Hortolândia, Paulínia e Valinhos. O perfil epidemiológico da DM na região foi analisado como em uma área única. São cidades conurbadas e possuem estreitas relações comerciais, econômicas e culturais, com intenso trânsito de pessoas entre elas. Situam-se no nordeste do Estado de São Paulo, possuindo ao redor de 1,2 milhões de habitantes. ${ }^{5}$

Considera-se pequena a proporção de subnotificações de casos na região devido à boa cobertura da investigação pelo sistema de vigilância epidemiológica dos municípios. Esta rotina é facilitada pelo empenho das equipes de saúde, pela gravidade do quadro clínico, pela necessidade de internações e 
pelas medidas de controle para evitar casos secundários. As mortes pela DM de residentes em Campinas foram verificadas no Banco de Óbitos Municipal, onde se verificou que nenhum caso de morte deixou de ser notificado.

O critério para definição de caso foi clínico e laboratorial, segundo padronização da Secretaria Estadual de Saúde de São Paulo. Isto é, cultura positiva do líquido céfalo-raquidiano ou do sangue, identificação de partículas de antígeno na imunoeletroforese cruzada ou na aglutinação pelo látex do líquor ou do soro, visualização do diplococo Gram negativo no exame bacterioscópico do líquor e presença de meningite com citoquímica do líquor compatível e/ou a presença de petéquias associadas com quadro infeccioso. ${ }^{9}$

Os exames laboratoriais para confirmação dos casos de DM foram realizados pelo Instituto Adolfo Lutz (IAL) regional de Campinas, designado Centro de Referência Nacional para Meningite (CRNM). O IAL recebeu as amostras para exame, provenientes de hospitais públicos e privados da área de abrangência do estudo. Para o diagnóstico laboratorial associou-se métodos sorológicos (imunoeletroforese cruzada e látex) aos bacteriológicos clássicos usados de rotina para investigação das meningites. ${ }^{9,10}$ Exames de maior complexidade como sorosubtipagem e determinação do multilocus enzimático foram realizados pelo IAL Central São Paulo.

Foram analisados dados secundários das fichas de investigação do Sistema de Vigilância Epidemiológica e do banco de dados Sinan (Sistema de Informações de Agravos de Notificação) da Direção Regional de Saúde - DIR-XII/ Campinas - SES/SP. Coletaram-se as seguintes informações: ano de ocorrência, faixa etária, cidade de moradia, forma clínica, época do ano, sorogrupo, sorotipo e subtipo da Neisseria meningitidis. Analisaram-se fatores possivelmente relacionados ao risco de morrer pela doença meningocócica. O critério diagnóstico foi também examinado como um indicador indireto da qualidade de serviço e da gravidade da doença.

As variáveis codificadas foram digitadas e analisadas em banco de dados do software Epi Info. ${ }^{4}$ Foi utilizado o teste qui-quadrado com correção de Yates, assumindo o nível de significância $\alpha=5 \%$ e odds ratio para a comparação de variáveis, considerando $95 \%$ o intervalo de confiança (IC). O software Statistica ${ }^{14}$ foi utilizado para a análise multivariada de regressão logística.

\section{RESULTADOS}

Nota-se aumento global do coeficiente de incidência da DM em quase todo o período, sendo os anos de
1995 e de 1996 os que apresentaram maior pico de incidência da infecção, e os anos de 1996 e de 1997 foram os de maiores incidências anuais, respectivamente 6,1 e 4,7 por 100.000 habitantes (Figura).

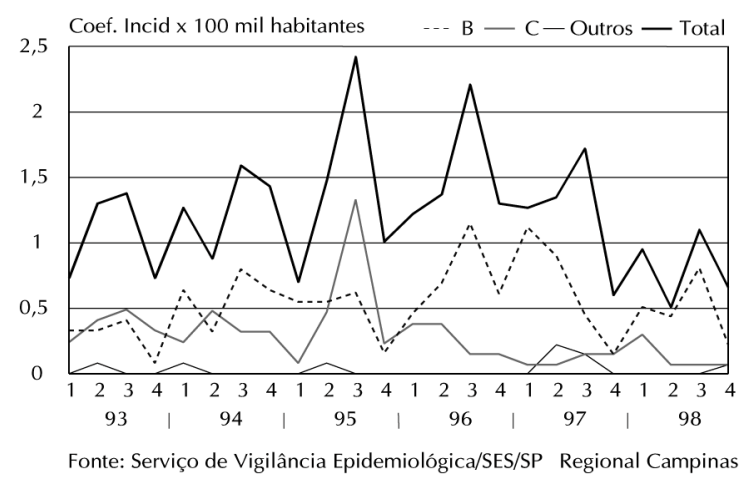

Figura - Coeficiente de incidência da DM (por 100.000 hab.) por Sorogrupo Região de Campinas (SP) - 1993 a 1998.

Do total de 375 casos estudados no período, foi identificado o sorogrupo do meningococo em $263(70,1 \%)$, observando-se predomínio do B (166 casos; 63,1\%), seguido pelo C (89 casos; 33,8\%). Em 187 casos (49,9\% do total) foi possível identificar além do sorogrupo, o sorotipo e o subtipo da $N$. meningitidis. Assim, 92 casos (48,9\%) foram da cepa B:4:P1.15 e $32(17,1 \%)$ da cepa C:2b:P1.3.

Observou-se que dos 92 casos pelo sorogrupo B sorosubtipados, $72,8 \%$ foram do sorotipo 4 e subtipo P1.15, apesar da circulação, no período, de outros 25 sorotipos e subtipos. Por outro lado, associados ao sorogrupo $\mathrm{C}$ foram isolados 16 sorotipos e subtipos, em 58 investigações, sendo que o $2 \mathrm{~b}: \mathrm{P} 1.3$ (32 casos) representou $56,1 \%$.

Em relação à distribuição etária dos casos, o grupo de maior risco da doença foi o de menores de um ano, com coeficiente de incidência de 46,1 por 100.000 habitantes, decrescendo com a idade até 0,9 por 100.000 habitantes em menores de 30 anos.

O sorogrupo B e, particularmente, a cepa B:4:P1.15 predominou em todas as faixas etárias, principalmente entre os menores de um ano $(17,4 \%$ das 25 cepas identificadas). Na faixa etária de 1 a 4 anos, a proporção foi semelhante entre os sorogrupos B e C, sendo que a cepa $\mathrm{C}: 2 \mathrm{~b}: \mathrm{P} 1.3$ foi mais freqüente nesta faixa etária $(56 \%)$ e na de 15 a 29 anos (25\%).

O padrão epidemiológico da DM nas cidades examinadas apresentou características homogêneas quanto à distribuição dos sorogrupos, com predomínio do B em todos os municípios (em média 69\% do sorogrupo B e $29 \%$ do C) e quanto ao coeficiente de letalidade, 
que em média foi de 19,0\%, com exceção da cidade de Valinhos, com oito casos e nenhum óbito.

\section{Letalidade da doença meningocócica}

A Tabela 1 mostra o coeficiente de letalidade da DM entre os sorogrupos na região de Campinas, apontando $22,2 \%$ nos 37 casos do sorogrupo B, 13,8\% nos 12 do sorogrupo $\mathrm{C}$ e $13,3 \%$ nos 15 casos não sorogrupados, no período estudado.

As duas cepas mais freqüentes da Neisseria meningitidis sorogrupadas e sorotipadas, B:4:P1.15 e C:2b:P1.3, apresentaram coeficientes de letalidade de $23,6 \%$ e $0 \%$, respectivamente. Os casos devidos à cepa B:4:P1.15 (92) apresentaram letalidade significativamente maior que todas as outras sorotipadas e subtipadas somadas (95) $\left(\chi^{2}=4,26 ; p=0,039\right)$.

A Tabela 2 apresenta os coeficientes de letalidade da DM segundo forma clínica e sorogrupos. A letalidade nos casos somente com meningite foi de $3,4 \%$, enquanto nos casos de meningococcemia pura foi de $43,5 \%$.

A Tabela 3 chama a atenção para a maior freqüência de casos de septicemia sem meningite entre os "não sorogrupados" $(27,6 \%)$, comparando-se com os casos de meningococcemia pura entre o sorogrupo B $(6,8 \%)$ e o C $(9,6 \%)$. Porém, o coeficiente de letalidade do meningococo B, nestes casos $(72,7 \%)$, se mantém mais alto em todos os outros sorogrupos, inclusive nos não-sorogrupados $-33,3 \%(\mathrm{p}=0,037$ teste exato de Fisher bilateral) (Tabela 2).

A distribuição etária dos óbitos indicou maior letalidade após 30 anos de idade $(30,5 \%)$ e em menores de 1 ano (25\%), sendo que 66,2\% dos óbitos ocorreram em menores de 4 anos.

A letalidade entre 30 e 39 anos foi particularmente alta, 42,9\%. Uma investigação mais detalhada das fi-

Tabela 1 - Evolução dos coeficientes de letalidade (\%) e número de óbitos (.) da doença meningocócica por sorogrupos na região de Campinas, SP, 1993 a 1998.*

\begin{tabular}{ccccccrr}
\hline Sorogrupo & 1993 & 1994 & 1995 & 1996 & 1997 & 1998 & $\begin{array}{r}\text { Total } \\
\%(.)\end{array}$ \\
& $\%()$. & $\%()$. & $\%()$. & $\%()$. & $\%()$. & $()$. & $\begin{array}{r}18 \\
\%\end{array}$ \\
\hline B & $7,1(1)$ & $23,3(7)$ & $25(6)$ & $26,3(10)$ & $23,5(8)$ & $18,5(5)$ & $22,2(37)$ \\
C & $5,6(1)$ & $12,5(2)$ & $7,4(2)$ & $21,4(3)$ & $80(4)$ & $0(0)$ & $13,8(12)$ \\
Não sorogrupados & $16,7(3)$ & $5,6(1)$ & $10(2)$ & $21,4(6)$ & $15(3)$ & $0(0)$ & $13,3(15)$ \\
Outros & $0(0)$ & $100(1)$ & $0(0)$ & $0(0)$ & $0(0)$ & $0(0)$ & $12,5(1)$ \\
\hline Total & $9,8(5)$ & $16,9(11)$ & $13,9(10)$ & $23,8(19)$ & $23,8(15)$ & $11,4(5)$ & $17,3(65)$ \\
\hline
\end{tabular}

*Foram incluídos como "não-óbito" os de evolução ignorada (7)

Fonte: Vigilância Epidemiológica - Secretaria de Saúde do Estado de São Paulo - Regional de Campinas

Tabela 2 - Coeficiente de letalidade (\%), número de óbitos(.) por sorogrupo da N eisseria meningitidis e forma clínica da doença meningocócica na região de Campinas, 1993 a 1998.*

\begin{tabular}{ccccc}
\hline $\begin{array}{c}\text { Sorogrupo } \\
\%(.)\end{array}$ & Só meningite & $\begin{array}{c}\text { Meningococcemia } \\
\text { com meningite } \\
\%(.)\end{array}$ & $\begin{array}{c}\text { Meningococcemia } \\
\text { sem meningite } \\
\%(.)\end{array}$ & Total \\
\hline B & $\%()$. & $30,2(26)$ & $72,7(8)$ & $22,2(37)$ \\
C & $4,6(3)$ & $19,1(9)$ & $37,5(3)$ & $13,8(12)$ \\
Outros** & $0(0)$ & $0(0)$ & $0(0)$ & $12,5(1)$ \\
Não sorogrupados & $14,3(1)$ & $11,1(6)$ & $33,3(9)$ & $13,3(15)$ \\
\hline Total & $0(0)$ & $21,8(41)$ & $43,5(20)$ & 65 \\
\hline
\end{tabular}

*O s casos com evolução ignorada (7) foram incluídos como não-óbito

**O utros sorogrupos são - W 135, Y e $29 \mathrm{E}$

Tabela 3 - Freqüência da forma clínica da doença meningocócica entre os sorogrupos da Neisseria meningitidis, região de Campinas, SP, 1993 a 1998.*

\begin{tabular}{|c|c|c|c|c|c|c|c|}
\hline \multirow[t]{2}{*}{ Sorogrupo } & \multicolumn{2}{|c|}{ Só meningite } & \multicolumn{2}{|c|}{$\begin{array}{l}\text { Meningococcemia } \\
\text { com meningite }\end{array}$} & \multicolumn{2}{|c|}{$\begin{array}{l}\text { Meningococcemia } \\
\text { sem meningite }\end{array}$} & \multirow{2}{*}{$\begin{array}{l}\text { Total } \\
\text { (.) } 100 \%\end{array}$} \\
\hline & $\%$ & (.) & & & & $()$. & \\
\hline$\stackrel{\stackrel{B}{C}}{\text { N }} \underset{\text { Outros** }}{*}$ & $\begin{array}{l}40,1 \\
33,7 \\
87,5 \\
17,4\end{array}$ & $\begin{array}{l}(65) \\
(28) \\
(7) \\
(17)\end{array}$ & $\begin{array}{l}53,1 \\
56,6 \\
12,5 \\
55,1\end{array}$ & $\begin{array}{l}(86) \\
(47) \\
(1) \\
(54)\end{array}$ & $\begin{array}{l}6,8 \\
9,6 \\
0,0 \\
27,6\end{array}$ & $\begin{array}{l}(11) \\
(8) \\
(0) \\
(27)\end{array}$ & $\begin{array}{c}162 \\
83 \\
8 \\
98\end{array}$ \\
\hline Total & & & & & & & 351 \\
\hline
\end{tabular}

*Não foram considerados 24 casos sem informação sobre a forma clínica

**O utros sorogrupos são - W 135, Y e $29 \mathrm{E}$ 
Tabela 4 - Fatores de risco para a letalidade da doença meningocócica na região de Campinas, SP, 1993 a 1998 (análise de regressão logística).

\begin{tabular}{|c|c|c|c|c|c|}
\hline $\begin{array}{c}\text { Variáveis } \\
\text { independentes }\end{array}$ & $\begin{array}{l}\text { Coef. de } \\
\text { letalidade } \\
(\%)\end{array}$ & $\begin{array}{c}\text { OR } \\
\text { Bruto** }\end{array}$ & $\begin{array}{c}\text { IC } \\
(95 \%)\end{array}$ & $\begin{array}{c}\text { OR } \\
\text { Ajustado*** }\end{array}$ & $\begin{array}{c}\text { IC } \\
(95 \%)\end{array}$ \\
\hline $\begin{array}{l}\text { Meningococcemia } \\
\text { Meningite pura } \\
\text { Idade }<1 \text { ano } \\
\text { Idade } 1 \text { a } 4 \\
\text { Idade } 5 \text { a } 29 \\
\text { Idade }>30 \\
\text { Sorogrupo B } \\
\text { O utros sorogrupos* } \\
\text { Não sorogrupados }\end{array}$ & $\begin{array}{r}26,1 \\
3,4 \\
25,0 \\
18,1 \\
8,3 \\
34,4 \\
22,2 \\
13,7 \\
13,3\end{array}$ & $\begin{array}{r}10,02 \\
0,10 \\
0,99 \\
1,07 \\
0,31 \\
2,87 \\
1,83 \\
0,71 \\
0,64\end{array}$ & $\begin{array}{c}3,32-33,4 \\
0,04-0,28 \\
0,54-1,83 \\
0,59-1,93 \\
0,51 \\
1,21-6,74 \\
1,03-3,26 \\
0,35-1,43 \\
0,34-1,19\end{array}$ & $\begin{array}{c}13,88 \\
1,0 \\
2,95 \\
2,22 \\
1,0 \\
6,42 \\
2,33 \\
1,48 \\
1,0\end{array}$ & $\begin{array}{c}4,68-42,13 \\
- \\
1,55-5,63 \\
0,63-2,37 \\
- \\
2,32-17,80 \\
1,14-4,79 \\
0,62-3,52 \\
-\end{array}$ \\
\hline
\end{tabular}

*O utros sorogrupos: C, W 135, 29E e Y

**O R (O dds Ratio) bruto - análise univariada (Kornelisse ${ }^{8}$ )

***O R ajustado pelo modelo da regressão logística ${ }^{31}$

IC = Intervalo de Confiança

chas epidemiológicas destes casos (21) e de óbitos (9) identificou meningococcemia, sorogrupo B, em todos eles, sendo 7 entre 9 casos residentes no município de Campinas.

A análise de regressão logística, cuja variável dependente foi a ocorrência do óbito, mostrou associação estatística da letalidade com as seguintes variáveis: presença da meningococcemia, com ou sem meningite; faixas etárias de maiores de 30 anos e menores de um ano; e casos devidos ao sorogrupo B (Tabela 4). O sorogrupo C, os outros e os não sorogrupados não se associaram ao óbito, na análise univariada e multivariada.

O período do ano de ocorrência da doença não influenciou a incidência de mortes mesmo em épocas de maior risco de adoecer. $\mathrm{O}$ odds ratio bruto entre os casos ocorridos nos meses de abril a setembro foi de 1,006 (IC 0,31-3,32). Os sorotipos e subtipos não foram analisados no modelo, pois dos 375 casos notificados, 50,1\% não dispunham desta informação.

\section{DISCU SSÃO}

A doença meningocócica na região de Campinas tem apresentado picos de incidência com características sazonais no período de 1993 a 1998 e tendência à queda em 1997, possivelmente relacionada ao impacto da campanha de vacinação contra o meningococo A e C em 1996, em alguns municípios da região. O comportamento da doença seguiu padrão da Grande São Paulo, onde desde 1988 observou-se maior prevalência da cepa B:4:P1.15, responsável pela continuidade da epidemia de DM naquela localidade. ${ }^{11}$ Os maiores coeficientes de incidência foram registrados entre menores de 4 anos, particularmente no primeiro ano de vida, decrescendo com a idade, enquanto a letalidade apresenta-se mais elevada nas faixas etárias extremas, conforme observado por outros autores. ${ }^{3,6,16}$
O coeficiente de letalidade é um indicador valioso no acompanhamento da $\mathrm{DM}$, podendo ser utilizado com maior freqüência nos sistemas de vigilância epidemiológica, municipais e regionais. Além disso, pode direcionar investigações mais aprofundadas e facilitar a atuação mais incisiva na prevenção de mortes.

$\mathrm{Na}$ região, esse coeficiente variou no decorrer dos anos, mostrando um aumento expressivo em $1996 \mathrm{e}$ em 1997 (23,8\%). Coincidiu com as maiores incidências do sorogrupo B, com altos percentuais de meningococcemia e com menor investigação etiológica dos casos. Aqueles anos também apresentaram os maiores índices de critério clínico para diagnóstico definitivo da DM, ao redor de $18,8 \%$.

A maior freqüência desse critério indica a insuficiente investigação do caso e o menor rigor diagnóstico justamente entre os doentes com meningococcemia sem meningite e mais graves. Nos casos de evolução fulminante, muitas vezes não há tempo hábil de acometimento meníngeo, ou mesmo impossibilidade de investigação adequada devido à urgência e ao limitado tempo para as tentativas de manejo do choque séptico, além da possibilidade destes casos não sorogrupados estarem encobrindo quadros bacterianos ou virais de outras etiologias.

A análise multivariada indicou a presença de meningococcemia, a faixa etária (maiores de 30 anos e menores de 1 ano) e o isolamento do sorogrupo B, associadas estatisticamente com o óbito, como apontado também por outros autores. ${ }^{8,16}$ Embora os coeficientes de letalidade tenham registrado a gravidade maior da doença nas faixas etárias extremas, os coeficientes de incidência mostraram valores decrescentes com a idade, como observado por outros autores. ${ }^{3,6,16}$

Ao se comparar os coeficientes de letalidade da DM na região de Campinas com países desenvolvidos, os valores encontrados no presente estudo mos- 
traram-se elevados. Os países escandinavos registraram coeficientes mais baixos nas últimas décadas. Como exemplo, na Suécia, a letalidade foi de $10 \%$, nos anos de 1987 a 1989. Na Noruega, ainda de grande importância epidemiológica, a DM apresentou sua maior letalidade no ano de 1994, com $16 \%$, sendo ao redor de $11 \%$ na década de 80 . A Finlândia, no final da década de 80 , notificou pequena letalidade, ao redor de 7,9\%. Na Islândia, a letalidade foi ao redor de $16 \%$ entre os 132 casos notificados nos anos de 1975 e de 1994 . $^{6,12,13,15}$

A partir de 1994 nota-se, na região, que a letalidade do sorogrupo B permaneceu estável e alta, porém, o sorogrupo C, os não sorogrupados e outros (Y, W135 e 29E) apresentaram grandes flutuações, mostrando instabilidade do indicador, em parte relacionada ao pequeno número absoluto de casos (Tabela 1). Ao contrário, no Canadá, a letalidade pelo sorogrupo C $(14,5 \%)$ suplantou a do sorogrupo B (6\%), sendo a cepa TE 15 identificada como a de maior virulência $(17,8 \%) .{ }^{16}$

A sazonalidade dos casos não influenciou a ocorrência do óbito, fato também observado em estudo canadense, mesmo com marcadas variações climáticas entre as estações. ${ }^{16}$

Muitas vezes, com os dados secundários disponíveis não é possível explicar a discrepância dessas variações. Análises mais apuradas implicam a implantação de instrumentos nos serviços de saúde para investigação sistemática dos óbitos no sentido de ampliar a compreensão desses eventos, das suas causas e das possibilidades de prevenção.

Os casos devidos ao sorogrupo B, mesmo apresentando menor percentual de formas septicêmicas, foram relacionados estatisticamente com o aumento da letalidade. Estes dados sugerem a maior virulência do sorogrupo B ou que, entre os casos de meningococcemia pura, possam existir casos de infecção por outros agentes etiológicos bacterianos ou virais entre os não sorogrupados, preservando o aumento do indicador de fatalidade da doença. Consta- ta-se a necessidade de maior especificidade na investigação etiológica. Estudo mais detalhado sobre as cepas predominantes e as de maior letalidade foi dificultado devido ao baixo índice de sorotipagem e de subtipagem $(50,1 \%)$, inviabilizando uma análise mais consistente sobre virulência e a comparação com o perfil epidemiológico de outras regiões.

Devido a variações na epidemiologia da doença e na sua distribuição temporal e espacial, o sorogrupo B apresentou-se como variável relacionada ao óbito. Porém, ocorrem variações cíclicas das cepas circulantes e da virulência. Vale lembrar que a confirmação do sorogrupo muitas vezes ocorre após o desfecho do caso particularmente em quadros fulminantes, não estando portanto esta informação disponível oportunamente como preditora. Alguns autores têm demonstrado a importância de se identificar as cepas predominantes e o aparecimento de novas, ligadas a variações do comportamento epidemiológico e à gravidade dos casos. ${ }^{1,16}$

Alguns autores identificaram outros preditores clínicos e laboratoriais do óbito, no decorrer da internação, e discutem a importância da qualidade da atenção médica e do cuidado intensivo na prevenção do óbito. ${ }^{3,8}$

Os dados secundários examinados no presente trabalho não permitem avaliar o papel da qualidade do atendimento na região. Certamente o diagnóstico precoce, $\mathrm{o}$ encaminhamento adequado dos pacientes e o tratamento correto influenciam o prognóstico dos casos.

\section{AGRADECIMENTOS}

Às equipes de vigilância epidemiológica municipal e estadual e do Instituto Adolfo Lutz, que compõem a Comissão Técnica para Controle da Doença Meningocócica na região de Campinas, pelo apoio e pelas discussões técnicas. Aos estatísticos: Andreia Ferreira e Helymar da Costa Machado, da Comissão de Pesquisa da Faculdade de Ciências Médicas da Unicamp, pela revisão estatística.

\section{REFERÊN CIAS}

1. Berrón S, Fuente LD, Martín E, Vásquez JA. Increasing incidence of meningococcal disease in Spain associated with a new variant of serogroup C. Eur J Clin Microbiol Infect Dis 1998;17:85-9.

2. Camargo MCC. Doença meningocócica no município de São Paulo, no período de 1979 a 1993: endemia e epidemia [dissertação]. São Paulo: Faculdade de Saúde Pública da USP; 1996.
3. Cubbels CL, García JJG, Martinez JR, O tín CL. Clinical data in children with meningococcal meningitis in a Spanish hospital. Acta Pediatr 1997;86:26-9.

4. Dean AG, Dean JA, Burton AH, Dicker RC. Epi Info, Version 5: a word processing database, and statistics program for epidemiology on micro computers [computer program]. Atlanta, Georgia: Center for Disease Control and Prevention; 1990. 
5. Fundação SEADE. São Paulo em dados: demografia do município de Campinas, Sumaré, Hortolândia, Paulínia e Valinhos - 1999 [on line]. Disponível em $<U$ RL:http.www.seade.gov.br>. [1999 set]

6. Iversen BG, Aavisland P. Meningococcal disease in Norway 1992-1995. Epidemiology and fatality. Scand J Infect Dis 1996;28:253-9.

7. Kemp B. Aspectos epidemiológicos e diagnóstico laboratorial da doença meningocócica no Município de Campinas/SP no período de 1988 a 1993 [dissertação]. São Paulo: Faculdade de Saúde Pública da USP; 1994.

8. Kornelisse RF, Hazelzet JA, Hop WCJ, Spanjaard L, Suur $\mathrm{MH}$, Van-Der- Voort E, et al. Meningococcal septic shock in children: clinical and laboratorial features, outcomes and development of prognostic score. Clin Infect Dis 1997;25:640-6.

9. Ministério da Saúde. Secretaria de Ações Básicas da Saúde. Divisão Nacional de Laboratórios de Saúde Pública. N ormas técnicas para o diagnóstico das meningites bacterianas. Brasília (DF): Centro de Documentação do Ministério da Saúde; 1986. (Série A: Normas e Manuais Técnicos, 32).

10. Rocha MMM, Esper MRNR, N eme SN, Medeiros MIC, Silva RRF, Arreaza ALV et al. Avaliação do diagnóstico laboratorial da doença meningocócica pelos laboratórios regionais do Instituto Adolfo Lutz. Rev Inst Adolfo Lutz 1999;58:33.
11. Sacchi CT, Lemos APS, Camargo MCC, Whitney AM, $M$ elles CEA, Solar CA. Meningococcal disease caused by $\mathrm{N}$ eisseria meningitidis serogroup $\mathrm{B}$ serotype 4 in São Paulo, Brazil, 1990 to 1996. Rev Inst Med Trop São Paulo 1998;40.

12. Sigurdardóttir $B$, Bjornsson $O M$, Jónsdóttir $E$, Erlendsdóttir H, Gudmundsson S. Acute bacterial meningitis in adults: a 20 year overview. Arch Intern Med 1997;157:425-30.

13. Spanjaard L, Bol P, De Marie S, Zanen HC. Association of meningococcal serotypes with the course of disease: serotypes $2 a$ and $2 b$ in N etherlands 1959-1981. J Infect Dis 1987;155:277-82.

14. StatSoft Inc. STATISTICA for Windows: computer program manual. Tulsa, O K: StatSoft; 1995.

15. Valmari P, Kataja M, Peltola $\mathrm{H}$. Invasive $\mathrm{H}$ aemophilus influenzae and meningococcal infections in Finland. Scand J Infect D is 1987;19:19-27.

16. Whalen CM, Hockin JC, Ryan A, Ashton F. The changing epidemiology of invasive meningococcal disease in Canada, 1985 through 1992. Emergence of a virulent clone of $\mathrm{N}$ eisseria meningitidis. JAMA 1995;273:390-4 